so-called 'permeability-transition pores"7.

Montero et al. then show that, as far as $\mathrm{Ca}^{2+}$ uptake is concerned, two different mitochondrial populations can be identified in intact cells. These two subpopulations differ not in terms of their intrinsic capacity to handle $\mathrm{Ca}^{2+}$, but in their localization with respect to the $\mathrm{Ca}^{2+}$ hotspots. Thus, mitochondria that are close to the regions of high cytosolic $\mathrm{Ca}^{2+}$ concentration show large increases in $\left[\mathrm{Ca}^{2+}\right]_{\mathrm{m}}$, whereas mitochondria in the other subpopulation take up barely any $\mathrm{Ca}^{2+}$. Most surprisingly, the authors show that the highly responsive mitochondrial subpopulation is the same whether the source of cytosolic $\mathrm{Ca}^{2+}$ is predominantly extracellular (with $\mathrm{Ca}^{2+}$ being transported into the cytosol through plasma-membrane $\mathrm{Ca}^{2+}$ channels) or intracellular (where $\mathrm{Ca}^{2+}$ is transported out of intracellular stores into the cytosol). In other words, Montero et al. suggest that, at least in chromaffin cells, there are functional 'units' composed of plasma-membrane and $\mathrm{ER} \mathrm{Ca}^{2+}$ channels, together with mitochondria, which are all recruited simultaneously during physiological cell activation.

Finally, Montero et al. look at the functional consequences of mitochondrial $\mathrm{Ca}^{2+}$ uptake. As would be predicted, inhibition of $\mathrm{Ca}^{2+}$ sequestration by mitochondria results in a dramatic increase in the amount of secretion of catecholamine more than five times more catecholamine is secreted from inhibited cells than from controls. This indicates that, in the inhibited cells, the lack of mitochondrial buffering of $\mathrm{Ca}^{2+}$ (which may result in either a greater increase in local cytosolic $\mathrm{Ca}^{2+}$ concentrations or a spreading of the $\mathrm{Ca}^{2+}$ signal to deeper cytoplasmic regions) may cause either the recruitment of catecholamine-containing secretory vesicles from distant sites, or more efficient fusion of already 'primed' secretory granules with the plasma membrane.

Montero et al.'s contribution prompts a number of exciting biological questions. For example, how much of the $\mathrm{Ca}^{2+}$ entering the cytoplasm from the extracellular medium and from the ER eventually ends up in the mitochondria? Which structure within the matrix is the actual $\mathrm{Ca}^{2+}$ buffer, and what is its capacity? What do mitochondria do under more physiological conditions - for example, when stimulated for only milliseconds rather than for seconds or minutes? Do these results relate only to chromaffin cells, or do they represent a general phenomenon? Before we can answer these questions, we will need further advances in methodology. Montero et al.'s results are averages from a cell population, and their time resolution is intrinsically low. A challenging task for the near future will be the development of methods as selective and quantitative as Montero et al.'s technique of targeting aequorin, but with signal intensities sufficiently high to be applicable to single-cell analysis. Finally, and ironically, we still have no clues to the identities of the molecular components of this physiologically important phenomenon - none of the mitochondrial $\mathrm{Ca}^{2+}$ carriers has ever been purified, and nor have their encoding genes been cloned. But we predict that the renewed interest in the mechanism of $\mathrm{Ca}^{2+}$ handling by mitochondria may eventually lead to the solution of this 30-year-old puzzle.

Tullio Pozzan is in the Department of Biomedical Sciences, University of Padova, Via Colombo 3,

35126 Padova, Italy.

e-mail:pozzant@civ.bio.unipd.it

Rosario Rizzuto is in the Department of

Experimental and Diagnostic Medicine, Section of

General Pathology, University of Ferrara, Via

Borsari 46, 44100 Ferrara, Italy.

e-mail:r.rizzuto@unife.it

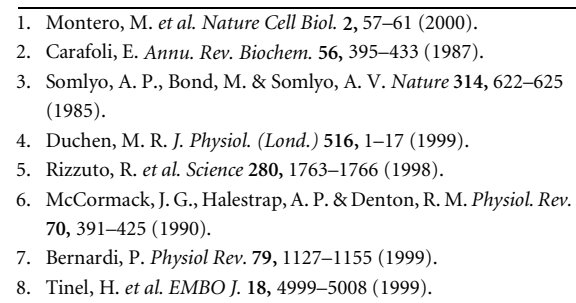

\title{
Hush, hush: the origin of telomeric silence
}

The ends of linear eukaryotic chromosomes - telomeres - consist of a specialized structure composed of a repetitive DNA sequence. Subtelomeric DNA, an operationally defined region adjacent to telomeres, is transcriptionally silent, a phenomenon known as telomeric position effect. As telomeres have been shown to localize near the nuclear envelope, these observations conjured up the question: is the location of telomeres responsible for their silence?

Writing recently in Nature (Nature 403, 108-112; 2000), Vincent Galy and colleagues propose an intriguing answer to this question. Galy et al. were studying budding yeast Mlp1 and Mlp2, proteins present on the nuclear side of the nuclear pore complex (NPC) - the structure through which RNAs and proteins shuttle between the nucleus and the cytoplasm. Surprisingly, mutations in the MLP1 and MLP2 genes did not affect nuclear transport. The morphology of the colonies of mutant strains, however, was strikingly similar to that of strains defective in DNA repair. Further analysis confirmed an unexpected role for the Mlp proteins in the repair of double-stranded breaks in DNA.

Ku70 is one of three components of DNA-dependent protein kinase (DNA-PK), a complex involved in double-strand-break repair. It is also necessary for telomeric silencing. So, Galy et al. tested whether Ku70 associated with telomeres in a strain lacking MLP2 ( $\Delta \mathrm{mlp} 2$ cells). Whereas, in wild-type cells, immunofluorescence identifying Ku70 was predominantly perinuclear, in $\Delta \mathrm{mlp} 2$ cells the signal was present throughout the nucleus. Galy et al. also detected a physical interaction between Mlp2 and Ku70; for example, only in the presence of Mlp2 was Ku70 found primarily associated with the nuclear matrix in fractionation studies. In $\Delta \mathrm{mlp} 1 \Delta \mathrm{mlp} 2$ cells, the spatial distribution of telomeric sequences changed from peripheral to central, and transcriptional
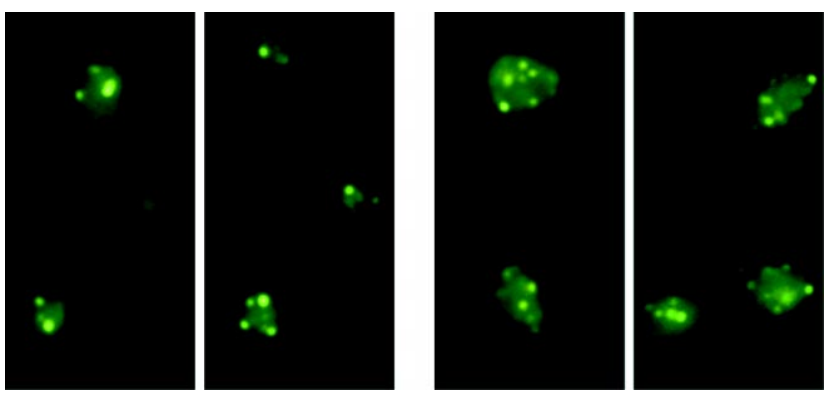

repression of a telomeric reporter gene was alleviated. Galy and colleagues identified an integral NPC component involved in RNA transport and pore clustering, Nup145, as the site of interaction of the Mlp proteins with the NPC. Deletion of Nup145 also resulted in the mislocalization of telomeres from the nuclear periphery (left panels) to the interior of the nucleus (right panels).

These results establish that the Mlp1 and Mlp2 proteins are required not only for the tethering of telomeres to the NPC, but also for efficient transcriptional repression at telomeres. The involvement of the Ku70 protein also hints at a role of the Mlp proteins in regulating DNA repair. The obvious next step is to determine whether an association between NPC core proteins, NPC proteins that extend into the nucleus and telomere-binding proteins constitutes a general mechanism of organizing telomeres into a topological domain, and recent studies seem to indicate that a similar process may operate in higher eukaryotic cells.

ANGELA EGGLESTON 